\title{
Characterization of the Decision Network for Wing Expansion in Drosophila Using Targeted Expression of the TRPM8 Channel
}

\author{
Nathan C. Peabody, Jascha B. Pohl, Fengqiu Diao, Andrew P. Vreede, David J. Sandstrom, Howard Wang, \\ Paul K. Zelensky, and Benjamin H. White \\ Laboratory of Molecular Biology, National Institute of Mental Health, National Institutes of Health, Bethesda, Maryland 20892
}

\begin{abstract}
After emergence, adult flies and other insects select a suitable perch and expand their wings. Wing expansion is governed by the hormone bursicon and can be delayed under adverse environmental conditions. How environmental factors delay bursicon release and alter perch selection and expansion behaviors has not been investigated in detail. Here we provide evidence that in Drosophila the motor programs underlying perch selection and wing expansion have different environmental dependencies. Using physical manipulations, we demonstrate that the decision to perch is based primarily on environmental valuations and is incrementally delayed under conditions of increasing perturbation and confinement. In contrast, the all-or-none motor patterns underlying wing expansion are relatively invariant in length regardless of environmental conditions. Using a novel technique for targeted activation of neurons, we show that the highly stereotyped wing expansion motor patterns can be initiated by stimulation of $\mathrm{N}_{\mathrm{CCAP}}$, a small network of central neurons that regulates the release of bursicon. Activation of this network using the cold-sensitive rat TRPM8 channel is sufficient to trigger all essential behavioral and somatic processes required for wing expansion. The delay of wing expansion under adverse circumstances thus couples an environmentally sensitive decision network to a command-like network that initiates a fixed action pattern. Because $\mathrm{N}_{\mathrm{CCAP}}$ mediates environmentally insensitive ecdysis-related behaviors in Drosophila development before adult emergence, the study of wing expansion promises insights not only into how networks mediate behavioral choices, but also into how decision networks develop.
\end{abstract}

\section{Introduction}

A primary function of the nervous system is to execute behavioral choices, that is, to alter an animal's behavior in response to changes in environment or internal demands. The neural mechanisms that underlie such choices have principally been investigated in reduced, invertebrate preparations, where work has focused largely on behaviors that are elicited by simple environmental stimuli and have motor outputs that are easily monitored in the absence of actual behavior (Kristan and Gillette, 2007). The introduction of genetically encoded effectors, which permit the targeted manipulation of specific neurons in intact animals (Miesenböck and Kevrekidis, 2005; Luo et al., 2008), is increasingly allowing the investigation of behavioral decisions that are based on more complex environmental stimuli and on neuromodulatory changes in internal state (Wu et al., 2003; Ada-

\footnotetext{
Received Sept. 5, 2008; revised Jan. 9, 2009; accepted Feb. 3, 2009.

This work was supported by the Intramural Research Program of the National Institute of Mental Health. We thank David Julius for providing the TRPM8 CDNA and John Ewer, Paul Taghert, and the Bloomington stock center for fly stocks. We appreciate early help with behavioral assays from Hailyn Nielsen and William Lemon. Special thanks to Grace Gray for editorial assistance and to Howard Nash for insightful comments and persistent insistence on clarity of thought.

Correspondence should be addressed to Benjamin H. White, National Institute of Mental Health, National Institutes of Health, 9000 Rockville Pike, Bethesda, MD 20892. E-mail: benjaminwhite@mail.nih.gov.

J. B. Pohl's present address: Graduate Program in Neuroscience, The University of Texas at Austin, 1 University Station, Austin, TX 78712.

D0I:10.1523/JNEUROSCI.4241-08.2009

Copyright $\odot 2009$ Society for Neuroscience $\quad$ 0270-6474/09/293343-11\$15.00/0
}

mantidis et al., 2007; Dickson, 2008; Yang et al., 2008). Because the new methods are easily deployed in genetic model organisms, such as Drosophila (Holmes et al., 2007), which are also amenable to developmental and comparative studies, they promise progress in addressing the broader issues regarding the development, evolution, and adaptive value of the processes and circuits underlying behavioral choice first articulated by Tinbergen (2005).

An extensively analyzed neuromodulatory circuit, for which physiological, developmental, and comparative data are available, is the network underlying ecdysis in insect molting (Ewer, 2005; Truman, 2005; Zitnan et al., 2007). This circuit orchestrates the motor patterns (the so-called "ecdysis sequence") needed to shed the old exoskeleton and expand and harden the new one. This sequence must be executed flawlessly, even in the absence of previous experience, and is therefore largely governed by endogenous factors, but often some components of the sequence are sensitive to external conditions to allow synchronization of hormonal release with environmental cues.

Such coordination is frequently required for the adapted ecdysis sequence used by adult insects to expand their wings, a process which in newly emerged flies requires a suitable perch (Cottrell, 1962; Fraenkel and Hsiao, 1965). If prevented from perching by perturbation or confinement, flies will choose to delay wing expansion for hours. The mechanisms underlying this choice are unknown, as are the neural determinants of the environmentally sensitive perch selection program. However, in Dro- 
sophila the network underlying wing expansion has been shown to include a set of neurons implicated in both larval and pupal ecdysis in Drosophila and in other insects (Gammie and Truman, 1997; Park et al., 2003; Clark et al., 2004; Luan et al., 2006). These neurons (i.e., $\mathrm{N}_{\mathrm{CCAP}}$ ) express crustacean cardioactive peptide, and a subset expresses the hormone bursicon (Dewey et al., 2004; Luo et al., 2005), which governs two motor patterns underlying wing expansion (Baker and Truman, 2002).

Here we combine environmental perturbations with targeted manipulations of $\mathrm{N}_{\mathrm{CCAP}}$ activity to show that environmental factors prolong the perch selection program and delay activation of the expansion program. We also introduce a method for acutely activating neurons by temperature decrements to demonstrate that $\mathrm{N}_{\mathrm{CCAP}}$ activation is sufficient to abrogate environmental inhibition and elicit the expansion program.

\section{Materials and Methods}

\section{Fly culture/crosses}

All flies were grown on corn meal-molasses medium and maintained at $25^{\circ} \mathrm{C}$ in a constant $12 \mathrm{~h} \mathrm{light/dark} \mathrm{cycle.} \mathrm{Wild-type} \mathrm{flies} \mathrm{(Canton-S} \mathrm{strain)}$ were from the Bloomington Stock Center (Indiana University, Bloomington, IN). The Gal4 driver lines used in this study were generous gifts of the following: $y w$; CCAP-Gal4; + and $w$; +; CCAP-Gal4 (Park et al., 2003, John Ewer); w; c929-Gal4; + (O'Brien and Taghert, 1998, Paul Taghert). The elav ${ }^{\mathrm{cl} 55}$-Gal4;+;+, yw; $2 \times$ UAS-EGFP; $2 \times$ UAS-EGFP, and $1 \times, 2 \times$, and $3 \times$ EKO lines, which carry one, two, and three copies of the EKO transgene have been described previously (White et al., 2001).

\section{Generation of UAS-TRPM8 constructs and fly lines}

The coding sequence of the TRPM8 cDNA (McKemy et al., 2002) was amplified by PCR using primers that introduced an EcoRI restriction site and an optimized translation initiation motif [with sequence CAAA, Cavener (1987)] immediately before the ATG start codon and a KpnI restriction site just after the stop codon. The amplified fragment was then subcloned into the pUAST plasmid for P-element transformation, using the unique EcoRI and KpnI restriction sites in the multiple cloning site. P-element injections and isolation of transformants was performed by Genetic Services. Flies with inserts of UAS-TRPM8 on the second ( $y w$; $C 4-D ;+)$ and third ( $y w ;+$; $4-A$ and $y w ;+; C 1-A 2)$ chromosomes were used in this study, separately or in combination.

\section{Behavioral observations and analysis}

Behavior of newly eclosed flies was observed by one of three methods as described in the Results. In all cases, flies were observed for at least $90 \mathrm{~min}$ after eclosion and wing expansion phenotypes were assessed at least $24 \mathrm{~h}$ after eclosion.

For observations in the low-perturbation condition, the vials used were either cultured at low density and cleared of any adults before observation of the first fly to eclose, or third instar larvae in the wandering stage were transferred individually to new culture vials to pupate and were observed after eclosion. Most observations were made by eye, although in a few cases flies were videotaped and behavior was scored from the video. The onset and offset of walking and abdominal flexion were scored to determine the three principal phases defined below.

For observations in the medium-perturbation condition, individual flies were transferred within 1-3 min of eclosion to a cylindrical chamber (1.4 $\mathrm{cm}$ diameter, $0.4 \mathrm{~cm}$ thick) formed by placing a plastic ring between two glass cover slides, which were held together by magnets glued to each slide. In some cases, an acetate sheet was attached to one of the glass slides to provide a better substrate for perching. Flies were videorecorded in this chamber using a Sony DCR-PC115 Digital Video Recorder, mounted on an Olympus SZX-12 stereomicroscope, with the video signal streamed to an external hard drive using a Firestore FS-1 (Focus Enhancements) for storage (see supplemental Fig. S1, available at www. jneurosci.org as supplemental material, for a full description of the setup). Videorecords for 40 animals were analyzed for sustained "state" behaviors (e.g., walking, grooming, abdominal contraction, proboscis extension, etc.) using Observer Video-Pro Version 5.0 software (Noldus
Information Techology). Detailed ethograms were constructed for 10 flies, scoring individual movements of the legs, wings, abdomen, and proboscis. Cibarial pumping, which appeared as pulses of light reflected from the base of the proboscis could not be resolved in all flies depending on their orientation relative to the camera.

For the high-perturbation condition, flies were transferred after eclosion into glass tubes $(0.3 \mathrm{~cm}$ diameter) plugged on each end to form a small chamber $0.7 \mathrm{~cm}$ in length. Flies confined within the tubes were videorecorded using a Sony HDR-FX7 digital videocamera, and records were scored for walking, grooming, abdominal flexion, abdominal contraction, and wing expansion.

The behavioral data in the text and in Figures $1 B$ and $5 A, B$ are presented as mean values \pm SEM. For box-and-whisker plots, the boxes represent the interquartile range of the data separated by the median value, and the "whiskers" extend to the minimum and maximum values of the data, with the exception of outliers. Outliers are defined as values that lie more than three times the interquartile range above the upper quartile value (i.e., top of the upper box). Data were analyzed using nonparametric statistics, due to significant heteroskedacity based on the Levene test. Comparisons of more than two groups were performed using the Kruskal-Wallis test, followed by repeated post hoc Mann-Whitney $U$ tests with Bonferroni corrections for multiple comparisons. Pairs of groups were compared with Mann-Whitney $U$ tests. All statistical analysis was performed with SPSS version 13.0.

\section{Definitions of behavioral phases}

Posteclosion behavior was divided into three phases, similar to those described previously in blowflies (Cottrell, 1962; Zdarek et al., 1984), defined by the following beginning and end points.

Phase I. From eclosion (or placement in the videorecording chamber) to first perch. A perch is defined as the cessation of walking for more than $5 \mathrm{~min}$. Flies sometimes commenced walking and reperched, but such episodes were typically brief compared with the initial walking bout.

Phase II. From first perch to the start of sustained abdominal flexion, a condition defined by the elongation and downward flexion of the abdomen. Although short pulses of abdominal flexion could be observed in phase II, sustained abdominal flexion typically lasted 10-15 min.

Phase III. From the onset to the cessation of abdominal flexion.

\section{Quantification of air swallowing}

Air swallowing was quantified by measuring the volume of air in the gut of fly. To do so, flies were briefly immersed in $100 \%$ ethanol to free the cuticle and bristles of any air, then pinned down through the head and anus in a Sylgard-treated culture dish filled with glycerol. The legs and wings were removed under a SZX-12 Olympus dissection microscope outfitted with a Nikon camera, and the gut was exposed by an incision extending up the midline of the fly. The air inside the gut was then released by gently tearing the gut membrane with forceps and, once liberated, formed a spherical bubble that rose slowly in the glycerol. This bubble was then photographed and its volume calculated from the value of the diameter measured using ImageJ software (W. S. Rasband, National Institutes of Health, Bethesda, MD, http://rsb.info.nih.gov/ij/) calibrated with a $2 \mathrm{~mm}$ micrometer. Typically, air volume was measured at the end of phase III or, in cases where this phase was absent, 90 min after the eclosion time.

\section{Testing UAS-TRPM8 and electrophysiology}

The efficacy of the TRPM8 channel was tested using a pan-neural driver

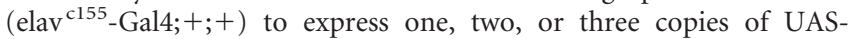
TRPM8. Up to 10 flies were placed in a large chamber (either a $35 \mathrm{~mm}$ culture dish or for the menthol experiments an empty food vial) and then either subjected to a temperature shift or exposed to saturating menthol vapor. The number of flies no longer standing (i.e., "flies down") was scored every $2.5 \mathrm{~min}$, typically from videorecords or in some cases by eye. Electrophysiological recordings from adult flight muscle were made as follows: Flies were anesthetized with $\mathrm{CO}_{2}$ just long enough to remove the wings and place them onto a temperature-controlled stage, where they were held in place with vacuum. To record UAS-TRPM8 activated motoneuron activity, electrolytically sharpened tungsten recording electrodes were placed in dorsal longitudinal muscles (DLM) and reference 
electrodes in the abdomen. Signals were amplified with Dagan IX2-700 (Dagan Corporation) or A-M Systems 1700 (A-M Systems) differential amplifiers. The temperature-controlled stage consisted of a hollow brass disc through which water of the appropriate temperature was circulated. Temperature changes of $>10^{\circ} \mathrm{C} / \mathrm{min}$ could be achieved by switching between two water baths maintained at target temperatures. Temperature was monitored with a thermocouple probe (T-type, Physitemp Instruments) placed adjacent to the fly, with the signal transduced by a temperature controller (CNI-3252-DC, Omega Engineering). Electrophysiological and temperature data were recorded using pClamp v. 8.2 (Molecular Devices).

\section{Menthol application}

Animals were exposed to saturating menthol vapors in plastic vials (94 $\mathrm{mm}$ long $\times 27 \mathrm{~mm}$ in diameter) as follows: flies were introduced into a vial capped by a foam plug in which menthol crystals were embedded. To test the effects of menthol on animals expressing UAS-TRPM8 panneuronally, 1-d-old adults were transiently immobilized by $\mathrm{CO}_{2}$ and permitted $1 \mathrm{~h}$ recovery in an empty vial before menthol exposure. Animals were observed and scored every two and a half minutes for $25 \mathrm{~min}$. To test the effects of menthol on wing expansion time, animals were collected within 5 min of eclosion and immediately transferred into a menthol-saturated vial for $15 \mathrm{~min}$. After $15 \mathrm{~min}$, the plug was gently replaced with one lacking menthol crystals. Individual flies were scored for wing expansion phenotypes at 1 min intervals.

\section{Hemolymph collection and immunoblotting}

For experiments on wild-type flies in the low-perturbation condition, hemolymph was extracted (as described below) from flies transferred into individual culture vials directly after eclosion and continuously monitored until they had spent $5 \mathrm{~min}$ in phases I, II, or III, or were at least 15 min past completion of phase III. For experiments in which flies expressing UAS-TRPM8 in $\mathrm{N}_{\text {CCAP }}$ had been subjected to a temperature shift in the high-perturbation condition, hemolymph was taken $1 \mathrm{~h}$ after the onset of the temperature shift (i.e., $45 \mathrm{~min}$ after return of the flies to $25^{\circ} \mathrm{C}$ ). Hemolymph was extracted in both cases by briefly anesthetizing flies with $\mathrm{CO}_{2}$, piercing the thorax with a needle and centrifuging as described previously (Luan et al., 2006). Collected samples were mixed with HE buffer (100 mM KCL, 20 mm HEPES-pH 7.5, 5\% glycerol, 0.5M EDTA, $0.1 \%$ Triton X-100) containing $2 \times$ Halt protease inhibitor (Pierce) and then frozen on dry ice. They were then thawed in equal portions of Laemmli sample loading buffer containing $5 \%$ $\beta$-mercaptoethanol, and boiled for 5 min before electrophoresis on a $12 \%$ Tris- $\mathrm{HCl}$ gel (Bio-Rad). Gels were transferred to $0.2 \mu \mathrm{m}$ nitrocellulose membranes using a Tris-Glycine- $20 \% \mathrm{MeOH}$ buffer, and immunoblotted. The primary rabbit anti-bursicon $\alpha$-subunit antibody (Luan et al., 2006) was used at a dilution of 1:5000 and a goat anti-rabbit secondary antibody $(10 \mu \mathrm{g} / \mathrm{ml}$, Pierce) at 1:2000. Blots were incubated with West Femto chemiluminescent substrate (Pierce) for 5 min before development on BioMax film (Eastman Kodak) for $10 \mathrm{~min}$.

\section{Immunohistochemistry and confocal analysis}

For analysis of whole-mount nervous system preparations, freshly emerged adults (i.e., within 15 min of eclosion) or stage P15i pharate adults (Bainbridge and Bownes, 1981) were dissected in PBS, and the excised nervous systems were fixed in $4 \%$ paraformaldehyde (Electron Microscopy Sciences) in PBS for $\sim 20$ min, followed by postfixation in $4 \%$ paraformaldehyde/PBS plus $0.5 \%$ Triton X-100 (Sigma) for $15 \mathrm{~min}$. Procedures for immunostaining were as previously described (Luan et al., 2006). Rabbit anti-TRPM8 antibodies (Novus Biologicals) were used at 1:200 dilution. Secondary antibodies (AlexaFluor 594 goat anti-rabbit from Invitrogen) were used at 1:500 dilution. Confocal imaging was performed using a Nikon C-1 confocal microscope. Z-series through either the brain or ventral nerve cord of each sample were acquired in 1 $\mu \mathrm{m}$ increments using a $20 \times$ objective unless otherwise noted, using 488 and $543 \mathrm{~nm}$ laser emission lines for EGFP and fluorophore excitation, respectively. Unless otherwise noted, the images shown are maximal projections of the volume rendered Z-stacks of confocal sections taken through the entire nervous system.

The consensus pattern of CCAP-Gal4>UAS-TRPM8 expression was determined by analyzing the intensity and frequency of labeling of identified CCAP-expressing neurons in multiple wholemount preparations. CCAP-expressing neurons in each preparation were identified in confocal sections by expression of UAS-EGFP and the intensity of TRPM8associated fluorescence was scored for each neuronal soma on a scale of $0-3$. The consensus intensity value $(I)$ for a given neuron was calculated by averaging all nonzero values for this neuron across preparations. The frequency $(v)$ with which a given neuron was labeled was calculated by dividing the number of preparations in which that neuron had a nonzero labeling intensity by the total number of preparations.

\section{Results}

\section{Environmental perturbation delays wing expansion}

The behavioral pattern required for wing expansion in Drosophila has been described previously as consisting of two principal phases: perch selection, in which the fly walks to identify a site for wing expansion, and expansion itself, during which the stationary fly swallows air and simultaneously contracts its abdomen to drive hemolymph (i.e., blood) into the wings (Baker and Truman, 2002). These behavioral phases are executed immediately after emergence from the pupal case (a process called eclosion) and also have been extensively characterized in blowflies (Zdarek et al., 1984). Genetic data demonstrate that the expansion phase is absent in Drosophila bearing null mutations in the rickets gene, which encodes the receptor for the hormone bursicon (Baker and Truman, 2002; Luo et al., 2005). However, apart from the observation of Kimura and Truman (1990) that fruit flies will delay wing expansion if forced to eclose into an empty puparium from which they cannot escape, there has been little investigation of how the behavioral programs underlying perch selection and wing expansion respond to environmental stimuli.

To analyze the behavioral consequences of different environmental conditions, we therefore investigated perch selection and wing expansion in wild-type flies in three different paradigms. In the first, which involved minimal perturbation, the flies were kept in the culture vial in which they had pupated (volume, $\sim 30 \mathrm{ml}$ ) and were observed by eye after eclosion. This condition required intermittently approaching the vial with a magnifying lens or gently manipulating the vial to optimize viewing as a fly moved. In the second paradigm, flies were gently transferred into a thin cylindrical chamber (volume, $0.616 \mathrm{ml}$ ) immediately after eclosion. This configuration (supplemental Fig. S1, available at www.jneurosci.org as supplemental material) was optimized for videomicroscopy to obtain comprehensive behavioral records, as described in Materials and Methods. Flies in the videochamber were exposed to a constant visual environment. In the third paradigm, flies were tapped into an even smaller cylindrical minichamber with cotton endplugs (volume, $4.9 \times 10^{-2} \mathrm{ml}$ ) and their behavior was videorecorded. In this paradigm, multiple chambers were monitored simultaneously and flies in adjacent chambers were exposed to each others' movements. The three paradigms thus differed in the degree of handling experienced by the fly after eclosion, the size of-and type of environment within-the chamber, and the visual environment to which the fly was exposed. We did not examine the contributions of each of these variables individually to the behavior of the fly, but each is likely to exert some effect on posteclosion behavior (J. Pohl, N. Peabody, and B. White, unpublished observations). In the following, we refer to the different paradigms as the "low-perturbation," "medium-perturbation," and "highperturbation" conditions to reflect the increasing degrees of stimulation and confinement experienced by the flies after emergence.

We found that the time required for flies to complete wing 
expansion varied considerably in the three different conditions, increasing progressively with the level of perturbation. In the low-perturbation condition, flies typically expanded their wings in approximately half an hour $(32.8 \pm 12.8 \mathrm{~min}, n=20)$, whereas in the medium- and high-perturbation conditions they took nearly three and four times this long $(83.8 \pm 30.7 \mathrm{~min}, n=40$ and $119.3 \pm 42.7 \mathrm{~min}, n=44$, respectively). Our preliminary analysis indicated that this difference was largely due to an extension of the perch selection phase, as flies in the smaller chambers persisted in walking for extended periods before finally engaging in wing expansion. This difference was most pronounced for flies in the high-perturbation condition, which sometimes walked for several hours. In addition, these flies frequently executed a distinct "digging" program (Fraenkel, 1935; Reid et al., 1987) when they encountered the cotton plugs at the ends of the chambers. They would attempt to push through the plug by expanding their ptilinum into the gap between the chamber wall and the plug while trying to propel themselves forward by abdominal, and sometimes leg, movements. Animals eclosing in the culture vials never displayed this behavior, and it was observed in the videochamber only on rare occasions when a small gap was accidentally left between the hard plastic ring and the glass plates that formed the chamber.

\section{Definition of posteclosion behavioral phases}

To quantify the differences between the behavioral patterns observed in the three paradigms, we required a baseline description of the post-emergence behavioral program and behavioral endpoints that could be used to define the phases. We therefore analyzed in detail the records of 10 flies observed in the videochamber where movements could be observed at the highest resolution. The pattern of behavior for all the flies was quite stereotyped, as previously observed by Baker and Truman (2002), and followed the progression shown in the ethogram of Figure $1 \mathrm{~A}$. Emerging flies, which have compact, folded wings (Fig. $1 \mathrm{Ai}$ ) first walked extensively and then perched. Sustained walking was rarely resumed after perching, although flies occasionally pivoted or walked short distances. While perched (Fig. 1Aii), the flies groomed intermittently and occasionally extended their abdomens or probosces. Eventually, the extension of both the proboscis and the abdomen became sustained, with the extended abdomen contracted radially and flexed downward (Fig. 1Aiiia). During this interval, the wings expanded, initially adopting a downwardly cupped shape, but gradually flattening while angled slightly away from the body axis. As the flattened wings were brought together over the body, the abdomen ceased to be flexed, although the proboscis often remained extended for some time. Grooming continued throughout the period of wing expansion, and persisted for some time afterward.

The overall behavioral pattern divided naturally into three principal phases, which we defined with respect to easily observable behavioral endpoints. As shown in Figure $1 B$, each phase is typically distinguished by a primary category of behavior. The perch selection phase, which we refer to as phase I, is dominated largely by walking and ends when the animal stops and then remains sedentary for at least $5 \mathrm{~min}$. The expansion phase, which we refer to as phase III, is defined by the onset and offset of tonic abdominal contraction. The proboscis also is extended during this phase and in favorably oriented flies $(n=7 / 10)$, the cibarial pump, which drives air swallowing, was seen to be active (Fig. $1 B)$. In the low-perturbation condition, flies dissected immediately after this phase had large bubbles in the abdomen (Fig. 1 Aiiib; Table 1), confirming the ingestion of air. Although expan- sion of the wings typically occurs during phase III, this event is thought to be mediated solely by movement of the blood into the wings rather than by exertions of wing-attached muscles. As described below, wing expansion can under some circumstances fail even when phase III and its associated motor patterns (i.e., abdominal contraction and air swallowing) appear normal. Interposed between phases I and III is a period dominated by grooming (Fig. $1 A, B$ ). We call this period, which also has been noted in blowflies (Cottrell, 1962), phase II. Twenty animals from the lowperturbation condition dissected during this behavioral phase were devoid of air in the gut, indicating that air ingestion is largely or completely restricted to phase III.

We also examined bursicon release as a function of behavioral phase for flies eclosing in their culture vials (i.e., the lowperturbation condition), where we could most readily collect sufficient numbers of similarly staged animals. Hemolymph samples collected from flies during phases I, II, and III, and $\sim 15$ min after the end of phase III were analyzed by Western blot (Fig. 1C). Bursicon was not detectable in the hemolymph of animals during phase I. It first appeared in phase II, and rose to its highest level during the expansion phase (phase III). Thus, in the lowperturbation condition, bursicon release appears to be initiated within $5 \mathrm{~min}$ of perch selection and to peak during wing expansion.

\section{Environmental perturbation extends the perch selection phase}

Having defined the behavioral phases, we compared their durations under the three environmental conditions (Fig. 2). This analysis confirmed that the differences in wing expansion time resulted primarily from a progressive increase in the duration of the perch selection phase. Mean phase I durations differed significantly between the three conditions ( $p<0.001$ by the KruskalWallis test), rising 20-fold between the low- and highperturbation conditions. In contrast, the mean duration of the expansion phase (III) was $\sim 15 \mathrm{~min}$ in all cases and did not differ significantly between perturbation conditions $(p>0.7$ by Kruskal-Wallis test). The stereotyped duration of phase III suggests that the motor patterns underlying expansion, once triggered, run for a fixed time. Bursicon is the likely trigger, and, as noted above, its release begins during phase II. Although phase II durations in the three different conditions were significantly different ( $p \leq 0.007$ in pairwise comparisons using the MannWhitney test), they showed no obvious trend with respect to the level of perturbation. The observed variability in phase II durations, both within any given condition and across conditions, suggests that the timing or rate of bursicon release may differ considerably among individual animals.

\section{The perch selection phase is insensitive to $\mathrm{N}_{\text {CCAP }}$ suppression}

The above data demonstrate that environmental perturbations delay wing expansion primarily by extending the perch selection phase. The perch selection program is thus modulated by the sensory pathways that mediate environmental perturbation, but it is possible that this modulation is indirect and requires the circuitry underlying the expansion program. For example, the expansion program may be the primary target of environmental modulation, and the perch selection program could simply be active until inhibited by initiation of the expansion program. If the expansion program never starts up, perch selection should continue indefinitely. To directly test the possible involvement of the expansion program circuitry, we examined the effects of in- 
A

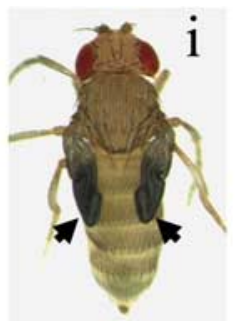

Phase I

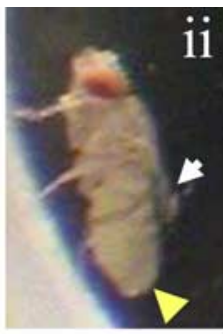

Phase II

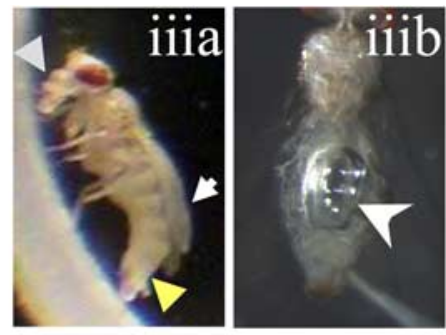

Phase III walking
grooming
hindleg
foreleg
midleg

Abd. flexion

Abd. elongation

proboscis ext. cibarial pumping
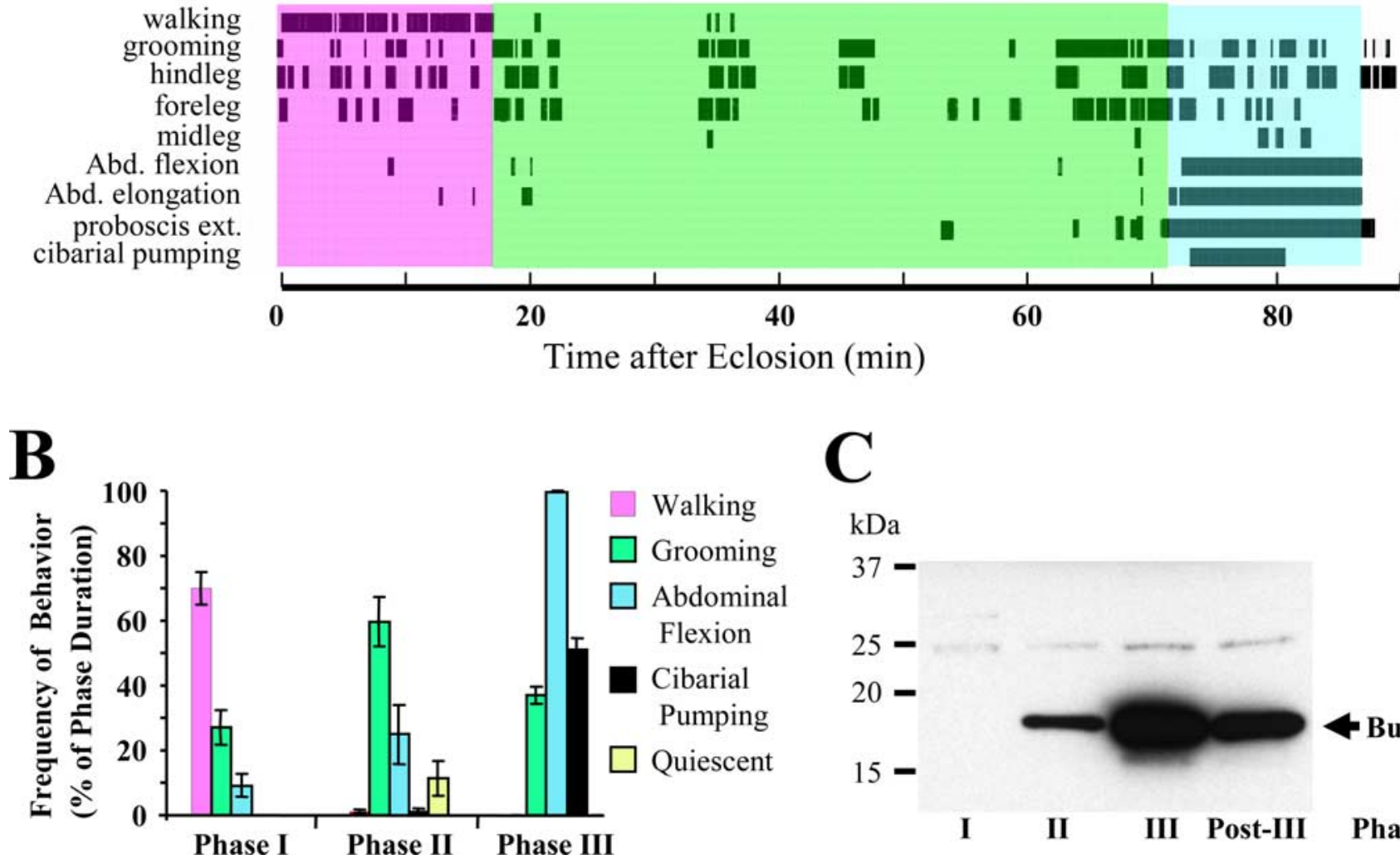

40

60

80
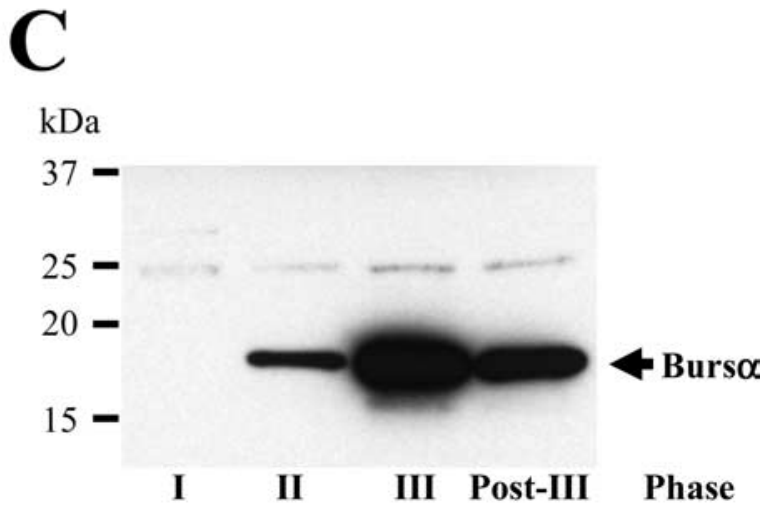

Figure 1. Posteclosion behavior in Drosophila consists of three phases with distinct patterns of behavior. $A$, Ethogram of a wild-type fly videotaped in the medium-perturbation condition. Bouts of walking, grooming, abdominal (Abd.) elongation and flexion, proboscis extension (ext.), and cibarial pumping can be resolved and scored in the detailed video records as indicated, as can individual movements of the three pairs of legs. The three principal phases of behavior are labeled and shaded, and an image of a representative fly in each phase is placed above the label. Phase I (magenta, $\boldsymbol{i}$ ) is distinguished principally by walking. At this time, the wings (black arrows) remain unexpanded. In phase II (green, ii), the fly is perched and grooms. Wings (white arrow) are still unexpanded and the abdomen (yellow arrowhead) is unflexed. In phase III (blue), the proboscis is extended (gray arrowhead, iiia) and the abdomen is elongated and flexed downward (yellow arrowhead) as the wings (white arrow) expand. During this phase, the fly swallows air, filling its gut with a large bubble (white arrowhead, iiib). $\boldsymbol{B}$, Bar graph indicating the average frequency of major state behaviors during each phase of flies in the medium-perturbation condition $(n=10)$. Shading is as indicated in the legend. Error bars represent SEM. C, Immunoblot of hemolymph samples collected $5 \mathrm{~min}$ into each phase (or $15 \mathrm{~min}$ after the termination of phase III for the last sample) from multiple flies. The blot was probed with an antibody against the bursicon $\alpha$-subunit. The position of the bursicon $\alpha$-subunit (Burs $\alpha$ ) is indicated by the arrow. Positions of molecular weight markers (in kDa) are indicated.

Table 1. Air-swallowing deficits correlate with the absence of abdominal contraction

\begin{tabular}{|c|c|c|c|c|}
\hline \multirow[b]{3}{*}{ Genotype } & \multicolumn{4}{|c|}{ Swallowed air (nl) } \\
\hline & \multicolumn{2}{|l|}{ End of phase III } & \multicolumn{2}{|l|}{ After $90 \mathrm{~min}$} \\
\hline & Males & Females & Males & Females \\
\hline Canton S (control) & $82 \pm 5(10)$ & $138 \pm 12(10)$ & $24 \pm 4(10)$ & $75 \pm 7(10)$ \\
\hline CCAP-Gal4 $>w^{1118}$ (control) & ND & ND & $38 \pm 6(10)$ & $106 \pm 6(10)$ \\
\hline CCAP-Gal4 $>1 \times$ UAS-EKO & $131 \pm 10(10)$ & $175 \pm 14(10)$ & $98 \pm 16(10)$ & $172 \pm 22(10)$ \\
\hline CCAP-Gal4 > 1× UAS-EKO (medium perturbation) & $131 \pm 16(8)$ & $196 \pm 36(7)$ & ND & ND \\
\hline CCAP-Gal4 > 2X UAS-EKO (no phase III) & ND & ND & $0 \pm 0(20)$ & $0 \pm 0(21)$ \\
\hline CCAP-Gal4 $>2 \times$ UAS-EKO (+ phase III) & $111 \pm 5(4)$ & $179 \pm 25(4)$ & $105 \pm 30(8)$ & $222 \pm 55(2)$ \\
\hline CCAP-Gal4 $>3 \times$ UAS-EKO & ND & ND & $0 \pm 0(9)$ & $0 \pm 0(10)$ \\
\hline rickets $^{4}$ & ND & ND & $0 \pm 0(10)$ & $0 \pm 0(10)$ \\
\hline
\end{tabular}

The amount of air (in nanoliters) swallowed by male and female flies of the indicated genotypes is shown together with the SEM. The volume of air found in the gut of dissected animals was determined as described in Materials and Methods. The number of animals assayed for each measurement is shown in parentheses. ND, Not determined. hibiting activity in $\mathrm{N}_{\mathrm{CCAP}}$ in the relative absence of environmental stimuli, i.e., in the low-perturbation condition.

We previously showed (Luan et al., 2006) that graded levels of $N_{\text {CCAP }}$ suppression can be achieved by using the CCAPGal4 driver to express increasing copy numbers of the UAS-EKO suppressor transgene. Expression of three copies of the UAS-EKO transgene (i.e., $3 \times$ UAS$\mathrm{EKO}$ ) in $\mathrm{N}_{\mathrm{CCAP}}$ completely blocks wing expansion and inhibits bursicon release into the hemolymph (Luan et al., 2006). As expected, we found that in the lowperturbation condition incremental 


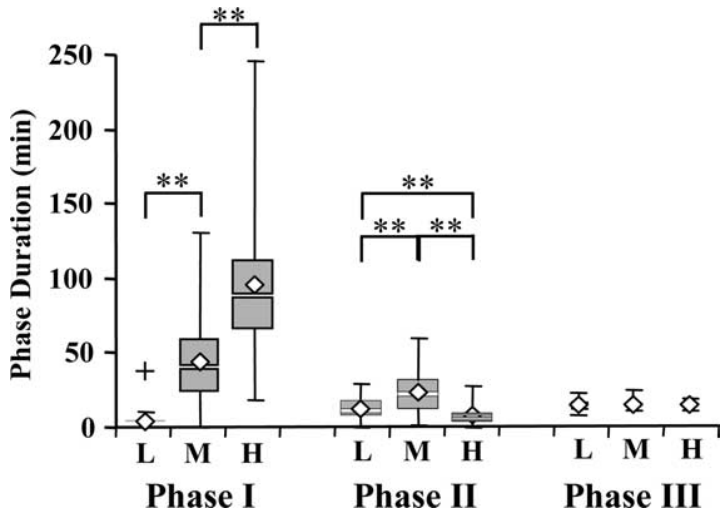

Figure 2. Environmental perturbation selectively extends the duration of the perch selection phase. Box-and-whisker plots show the distribution of phase durations for flies observed under the low (L)-, medium (M)-, and high (H)-perturbation conditions described in the text. Each graph shows the durations of one of the three principal behavioral phases. The duration of the perch selection phase (phase I) increases incrementally with perturbation level, while the expansion phase (phase III) is unaffected by environmental conditions. The grooming phase (phase II) does not vary systematically with perturbation. For each plot, the lower and upper boxes span the second and third quartiles of the data, with the division between them defining the median. The whiskers extend to the minimum and maximum values of the data except when outliers are present (see Materials and Methods), in which case the outliers are represented by individual plus signs. Diamonds represent mean values. Double asterisks indicate very significant differences $(p \leq 0.01)$ as determined by the Mann-Whitney test. For the low-, medium-, and high-perturbation conditions, $n=20,40$, and 44 , respectively.

$\mathrm{N}_{\text {CCAP }}$ suppression causes wing expansion deficits of increasing severity and frequency (Fig. 3A, top panel). Consistent with the known requirement for bursicon in expansion behaviors (Baker and Truman, 2002), suppression of $\mathrm{N}_{\mathrm{CCAP}}$ by $3 \times$ UAS-EKO completely eliminated both the tonic abdominal contraction that defines phase III (Fig. 3A, bottom panel) and air swallowing (Table 1; see also supplemental Movie S2, available at www. jneurosci.org as supplemental material). Despite the absence of the expansion phase, however, the duration of phase I in these animals was indistinguishable from that of the control flies (Fig. $3 B)$. Indeed, there was no significant difference in the length of phase I for any of the experimental groups $(~ p>0.2$ by KruskalWallis test), and almost all the flies completed perch selection in $<10$ min regardless of the level of $\mathrm{N}_{\mathrm{CCAP}}$ inhibition. Our results demonstrate that $\mathrm{N}_{\mathrm{CCAP}}$ neurons are not involved in the termination of perch selection, and therefore in the transition to phase II, but that they are essential for the subsequent activation of the expansion program.

$\mathrm{N}_{\text {CCAP }}$ activity regulates initiation of the expansion program The behavioral effects of $\mathrm{N}_{\mathrm{CCAP}}$ suppression have implications not only for the regulation of the perch selection program, but also for the mechanisms governing the wing expansion-related motor patterns. In this regard, the results for animals expressing $2 \times$ UAS-EKO are of particular interest. These flies can be divided into two distinct categories: Approximately half resembled flies expressing $1 \times$ UAS-EKO, which exhibited a robust expansion phase that included tonic abdominal contraction (Fig. 3A, bottom panel) and air swallowing (Table 1), while the rest resembled $3 \times$ UAS-EKO animals, which completely lack phase III behaviors (Fig. 3A, bottom panel; Table 1). This observation implies that there is a threshold level of $\mathrm{N}_{\mathrm{CCAP}}$ activity which must be exceeded if the expansion phase is to be initiated. Because the two behaviors required for wing expansion either occur or fail in
A

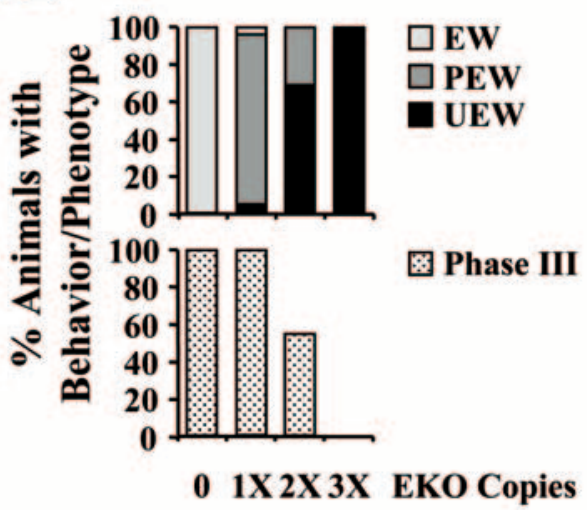

B

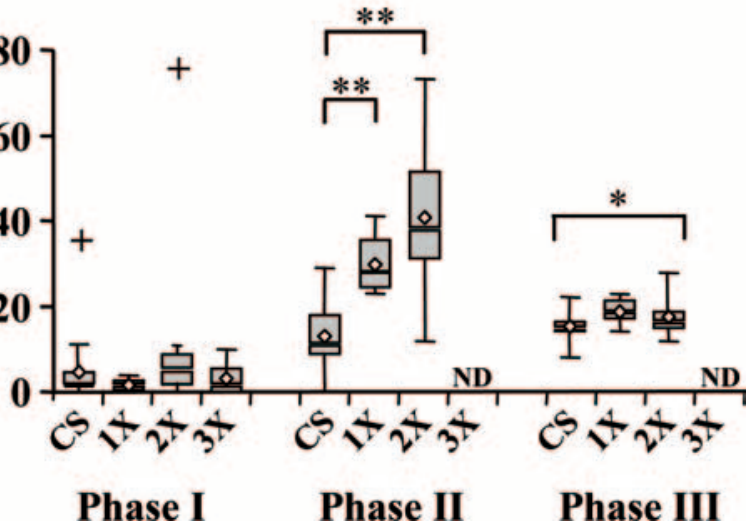

Figure 3. Graded suppression of $\mathrm{N}_{\text {CCAP }}$ incrementally inhibits wing expansion and slows or blocks entry into the expansional phase. $A$, Inhibition of $\mathrm{N}_{\text {CCAP }}$ in the low-perturbation condition by expression of one to three copies of the suppressor transgene, UAS-EKO, incrementally increases wing expansion deficits (top graph) and decreases the percentage of animals that execute phase III (bottom graph). EW, PEW, and UEW represent expanded, partially expanded, and unexpanded wing phenotypes, respectively. All data were taken from animals tested in the low-perturbation condition. $B$, Box-and-whisker plot showing the distribution of phase durations for animals expressing $1-3 \times$ UAS-EKO in the low-perturbation condition. Definitions are as described in the legend of Figure 2 . Animals expressing $3 \times$ UAS-EKO failed to enter phase III, and phase II was therefore of indeterminate length. Both phases are rendered as "not defined" (ND) for these animals. Phase II and phase III plots for animals expressing $2 \times$ UAS-EKO include data only from the 12 animals (of 25 total) that executed phase III. Also, the phase I data set for these animals included three outliers, only one of which is represented, because two of the animals walked for the entire observation period and therefore did not end phase I. Only a median value (and no mean) is therefore given for this plot. For $1 \times$ and $3 \times$ UAS-EKO conditions, $n=10$ and 6 , respectively. Plus signs and double asterisks are as defined in the legend of Figure 2. A single asterisk indicates a significant difference $(p<0.05)$ across conditions as determined by the Kruskal-Wallis test.

tandem, our results also imply that the two motor patterns share a common mechanism of initiation.

The common mechanism of initiation of the phase III motor patterns most likely reflects their shared requirement for bursicon (Baker and Truman, 2002), and their coincident failure at higher levels of $\mathrm{N}_{\text {CCAP }}$ suppression presumably results from impaired hormone release. Impaired bursicon release is also likely to underlie the observation that animals expressing $1 \times$ UAS-EKO, as well as approximately one-half of animals expressing $2 \times$ UASEKO, initiate phase III only after a significant delay, as reflected in the extension of phase II (Fig. 3B). Further work will be required to determine whether the extension of phase II, which appears to result from longer quiescent episodes between grooming bouts, is 
caused by the slowing of bursicon release or by its delay (or both), but the former possibility would conveniently explain the observed threshold in $\mathrm{N}_{\mathrm{CCAP}}$ activity for wing expansion. If EKOmediated suppression attenuates bursicon release, the threshold for $\mathrm{N}_{\mathrm{CCAP}}$ activity would simply reflect a threshold requirement for bursicon, with expansion triggered only when bursicon reaches a critical level.

A final implication of the results shown in Figure 3 is that $\mathrm{N}_{\mathrm{CCAP}}$ modulates not just the expansion program, but also the nonbehavioral processes necessary for wing expansion. This is again most obvious for flies expressing $2 \times$ UAS-EKO. Approximately half of these flies executed phase III (Fig. $3 A$, bottom panel), but none of these expanded their wings fully, and $70 \%$ did not expand them at all (Fig. $3 A$, top panel). In addition, most flies expressing $1 \times \mathrm{UAS}$-EKO in $\mathrm{N}_{\mathrm{CCAP}}$ only partially expanded their wings, despite executing normal phase III behavior. While we cannot rule out subtle behavioral deficits in the animals that failed to expand their wings, our results suggest that $\mathrm{N}_{\mathrm{CCAP}}$ regulates nonbehavioral processes involved in wing expansion, such as plasticizing the wing cuticle to render it pliable. These nonbehavioral processes may well be mediated by bursicon released into the hemolymph, as the blood-borne hormone is known to be required for somatic changes at the level of the wing cuticle (Reynolds, 1976, 1977; Dai et al., 2008). In contrast to motorpattern deficits resulting from $\mathrm{N}_{\mathrm{CCAP}}$ inhibition, which are all-ornone, wing expansion defects are often partial (Fig. $3 A$, top panel) and change in a graded manner. The nonbehavioral deficits associated with $\mathrm{N}_{\text {CCAP }}$ suppression thus appear to lack a threshold.

\section{Targeted expression of rat TRPM8 can be used to acutely activate neurons}

The suppression experiments described above indicate that perch selection normally terminates without input from $\mathrm{N}_{\text {CCAP }}$ and that the bursicon released from $\mathrm{N}_{\mathrm{CCAP}}$ during phase II initiates the expansion program. Initiation of expansion thus requires activation (or disinhibition) of $\mathrm{N}_{\mathrm{CCAP}}$, and these neurons are probable targets of modulation by circuits that mediate the perch selection program or environmental inputs. To determine whether $\mathrm{N}_{\text {CCAP }}$ neurons are the sole targets of such modulation, or whether downstream neurons that mediate the execution of the expansion program are also subject to regulation, we sought to artificially activate $\mathrm{N}_{\mathrm{CCAP}}$ during perch selection. We reasoned that if neurons downstream of $\mathrm{N}_{\mathrm{CCAP}}$ are normally inhibited during perch selection, either by the circuitry underlying this program or by negative environmental signals, activation of these neurons would fail to initiate the expansion motor patterns. If, on the other hand, artificial activation of $\mathrm{N}_{\mathrm{CCAP}}$ readily induced expansion, this would provide strong evidence that neural substrates of the expansion program downstream of $\mathrm{N}_{\mathrm{CCAP}}$ were unlikely regulatory targets of the circuits underlying perch selection and/or environmental inputs. To determine which of these two possibilities might be the case, and more generally to determine whether $\mathrm{N}_{\mathrm{CCAP}}$ activity is sufficient to initiate expansion behavior, we sought to acutely stimulate these neurons in posteclosion flies.

Efforts to use the light-sensitive Channelrhodopsin-2 protein (Nagel et al., 2003), which has been used successfully before in Drosophila for acute neuronal activation (Schroll et al., 2006; Suh et al., 2007), proved ineffective in newly emerged adults, perhaps due to poor light penetration through the cuticle or depletion of the retinal-A cofactor during metamorphosis. We therefore sought to develop an alternative, and simpler, system for stimulating neurons in the live animal. To this end, we generated trans- genic flies that can express the rat TRPM8 gene, which encodes a nonselective cation channel expressed in mammalian sensory neurons (McKemy et al., 2002; Peier et al., 2002). This channel is responsible for the sensation of mild cold (Colburn et al., 2007; Dhaka et al., 2007) and activates in response to decrements in temperature in the range of $\sim 25^{\circ} \mathrm{C}$ to $10^{\circ} \mathrm{C}$. The thermal sensitivity of the TRPM8 channel, which is compatible with the temperatures at which flies are normally active, makes it particularly suitable for manipulating activity in Drosophila. Because cold, unlike light, normally attenuates motor activity, neuronal activation using TRPM8 also has an advantage over optical techniques in minimizing the confounding behavioral effects of the activating stimulus.

We made transgenic fly lines that express the TRPM8 channel under the control of Gal4, and tested their efficacy by assaying for abnormal behavior when the channel was expressed panneuronally. Several lines expressed the TRPM8 channel at sufficiently high levels to give robust phenotypes (such as seizing, jumping, and eventually falling down) upon transfer to $15^{\circ} \mathrm{C}$ from room temperature. We combined the strongest inserts to make lines that bore from one to three copies of the UAS-TRPM8 transgene. As shown in Figure $4 A$, flies expressing UAS-TRPM8 pan-neuronally fell down quickly upon transfer to $15^{\circ} \mathrm{C}$, with animals expressing $3 \times$ UAS-TRPM8 displaying a more rapid and robust response. Electrophysiological recordings from flight muscles of these animals revealed sustained action potential generation in motoneurons in response a downward temperature ramp (Fig. $4 B$ ). This effect was readily reversible. Males typically showed greater sensitivity than females to a given temperature shift, as can be seen from the responses of animals expressing $2 \times$ UAS-TRPM8 shifted to $18^{\circ} \mathrm{C}$ (Fig. $4 \mathrm{C}$ ). At this temperature, $60 \%$ of males eventually fell over and could not right themselves, but none of the females did so, even after $10 \mathrm{~min}$. We also tested the sensitivity of flies expressing UAS-TRPM8 to menthol. Menthol potentiates the activity of TRPM8 by shifting its temperature sensitivity to more positive temperatures (McKemy et al., 2002). Accordingly, saturating menthol vapor administered to flies expressing UAS-TRPM8 pan-neuronally at room temperature $\left(24^{\circ} \mathrm{C}\right)$ caused the animals to seize and fall over as they did in response to cold (Fig. $4 D$ ). The response was slow and poorly penetrant with a single copy of the transgene, but most animals expressing $2 \times$ UAS-TRPM8 fell down within 5 min of menthol exposure and all were down by $10 \mathrm{~min}$.

\section{Activation of $\mathrm{N}_{\text {CCAP }}$ using TRPM8 is sufficient to initiate wing expansion}

To activate $\mathrm{N}_{\text {CCAP }}$ with stimuli of various magnitudes, we subjected flies expressing $1 \times$ and $2 \times$ UAS-TRPM 8 in $\mathrm{N}_{\mathrm{CCAP}}$ (see Fig. S3 for anti-TRPM8 immunostaining) to temperatures shifts of various magnitudes. Under conditions similar to our lowperturbation condition, newly eclosed flies were transferred into food vials at room temperature and then placed at temperatures ranging from $18^{\circ} \mathrm{C}$ to $12^{\circ} \mathrm{C}$. Even the mildest stimulus tested gave robust results: CCAP-Gal4 $>1 \times$ UAS-TRPM 8 animals shifted briefly to $18^{\circ} \mathrm{C}$ immediately after eclosion rapidly expanded their wings relative to controls bearing the $1 \times$ UAS-TRPM 8 transgene without the CCAP-Gal4 driver (Fig. 5A, left). Both the speed with which the experimental group expanded their wings and the low variance in expansion times implied strong and uniform stimulation of $\mathrm{N}_{\mathrm{CCAP}}$ in these animals, effects that were also observed when wing expansion was induced with a $15 \mathrm{~min}$ exposure to saturating menthol vapor at room temperature (Fig. $5 A$, right).

To determine whether strong induction of the perch selection 
program attenuated the ability of UASTRPM8 to drive wing expansion, we examined the response of flies in the highperturbation condition. Newly eclosed animals were placed in minichambers and exposed to a 15 min temperature shift from $24^{\circ} \mathrm{C}$ to $18^{\circ} \mathrm{C}$. We found that $\mathrm{N}_{\mathrm{CCAP}}$ activation by UAS-TRPM8 resulted in a substantial acceleration of wing expansion in all animals (supplemental Movie S4, available at www.jneurosci.org as supplemental material). On average, these animals took $25 \pm 3 \min (n=12)$ to expand their wings compared with $219 \pm 25 \mathrm{~min}$ $(n=10)$ for control flies bearing the $1 \times$ UAS-TRPM8 transgene, but lacking the CCAP-Gal4 driver (Fig. 5B). All behavioral phases were typically present in the experimental animals, although phase II was extremely brief. Most animals persisted in walking and digging through most of the 15 min temperature pulse, but then transitioned almost immediately into the expansion phase after perching. Consistent with our observations under other conditions, phase III was relatively uniform in duration and lasted on average $\sim 15 \mathrm{~min}$. All animals remained sedentary during wing expansion, indicating that execution of the expansion program potently inhibits perch selection behaviors. In addition to performing the expansion motor programs, animals expressing $1 \times$ UAS-TRPM8 in the high-perturbation condition displayed normal cuticle tanning after $\mathrm{N}_{\mathrm{CCAP}}$ activation (Fig. $5 C$, compare left, right). $\mathrm{N}_{\mathrm{CCAP}}$ activation thus appears to induce bursicon release into the hemolymph. We confirmed this directly by measuring hormone titers after the temperature shift by Western blot (Fig. $5 D)$.

Thus, under conditions that normally bias the animal to persist in perch selection behaviors, mild activation of $\mathrm{N}_{\mathrm{CCAP}}$ initiates hormone release and induces both the behavioral and physiological hallmarks of bursicon action. These results indicate that the neural pathways mediating perch selection and/or environmental input regulate the wing expansion program at, or above, the level of $\mathrm{N}_{\mathrm{CCAP}}$, and that neurons downstream of $\mathrm{N}_{\mathrm{CCAP}}$ are not targets of modulation. In addition, our results demonstrate that $\mathrm{N}_{\mathrm{CCAP}}$ neurons are not only necessary for wing expansion but also sufficient to drive this entire process.

\section{Discussion}

The introduction of genetically encoded effectors, which permit the targeted manipulation of neuronal activity in vivo, is increasingly facilitating neurobiological studies of behavioral choice in vivo, as exemplified by the work presented here. Using a new method for stimulating neurons in live animals, we demonstrate that the $\mathrm{N}_{\mathrm{CCAP}}$ network, first implicated in governing ecdysis, effects the fly's decision to expand its wings after eclosion. This decision is normally coupled to the decision to perch, which we demonstrate is based on evaluation of environmental variables. expression levels in these animals.

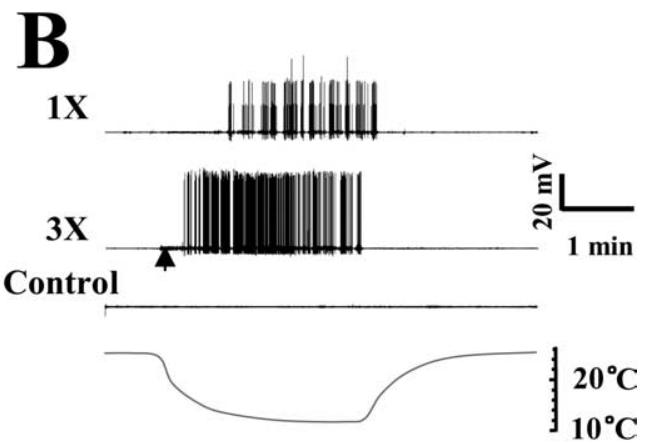

D

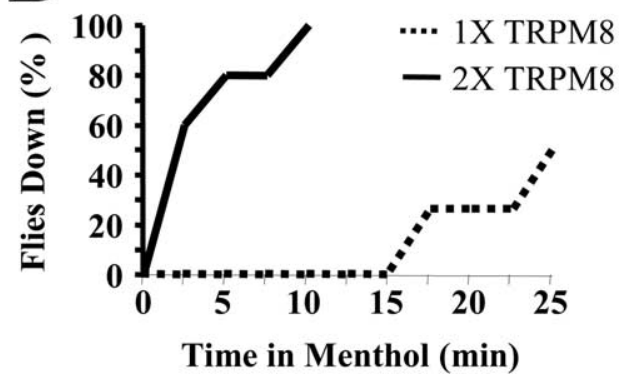

Time in Menthol (min)

Figure 4. UAS-TRPM8 acutely activates neurons in response to temperature decrements. One to three copies of a UAS construct expressing the rat cold-and-menthol receptor, encoded by the TRPM8 gene, were targeted to neurons using the pan-neural activity in flight muscles elicited by stimulation of innervating motoneurons. Animals expressing $1 \times$ (top trace) or $3 \times$ (middle trace) UAS-TRPM8 pan-neuronally were exposed to a 3 min temperature ramp from 23 to $12^{\circ} \mathrm{C}$ (bottom trace). The time of onset (a) UAS-TRPM8 (dotted line), but the rate is much faster in animals expressing $2 \times$ UAS-TRPM8 (solid line), consistent with higher

The postponement of wing expansion by the fly under adverse circumstances is thus a consequence of a value-based choice to prolong search behavior and the delayed activation of a command network responsible for the execution of the wing expansion decision.

\section{Perch selection is environmentally regulated}

As indicated in Figure 6, our results support a model in which the motor programs underlying perch selection respond primarily to environmental input. Although the environmental features monitored by the fly and the sensory channels that process them remain to be determined, it is clear from our experiments that flies assess conditions during phase I and assign longer search periods to more adverse environments. The observation that expansion is not deferred indefinitely, even under the highperturbation condition, suggests that the benefits of continued searching are weighed against the risks of further delay. How these risks, which may include predation and desiccation, are represented physiologically to encode the "value" of different environments is still unclear, but the work described here should facilitate their investigation. 


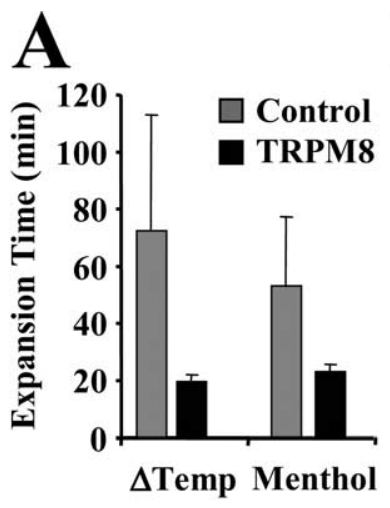

B
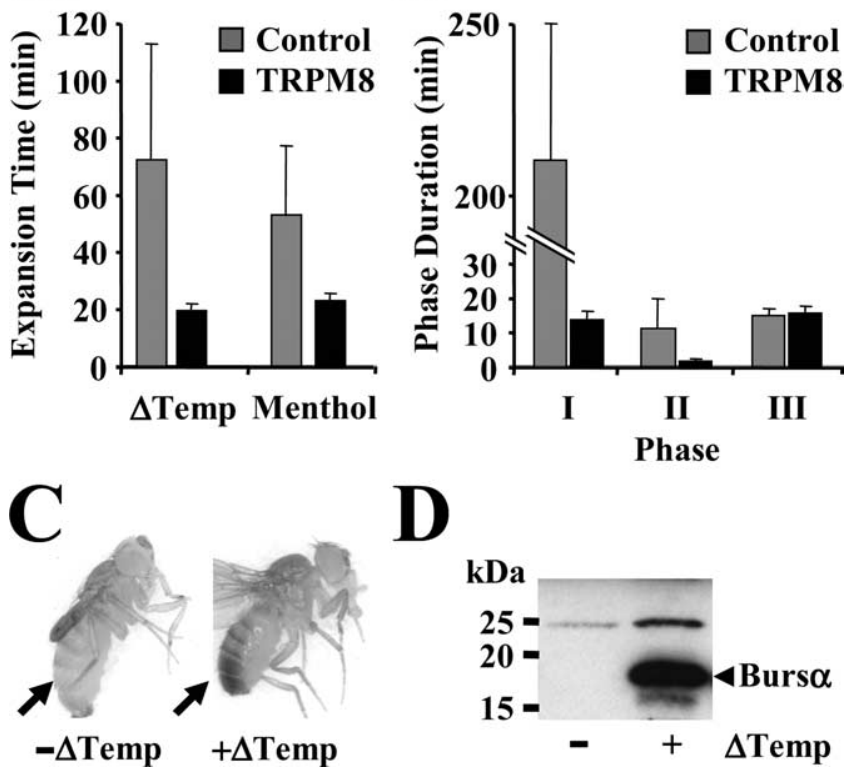

D

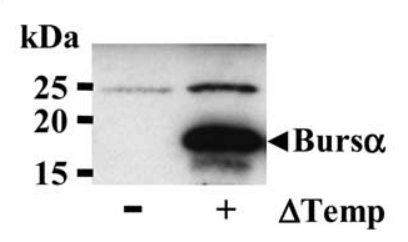

Figure 5. Stimulation of $\mathrm{N}_{\text {CCAP }}$ using UAS-TRPM8 drives rapid wing expansion and bursicon release in animals in the high-perturbation condition. $A$, Bar graphs show the time taken by flies expressing UAS-TRPM8 in $\mathrm{N}_{\text {CCAP }}$ to expand their wings after temperature shift to $18^{\circ} \mathrm{C}(\Delta$ Temp) or exposure to saturating menthol vapor (Menthol). In each case, the time taken by CCAPGal4 $>1 \times$ UAS-TRPM 8 animals $(n=31$ and 18 for the $\Delta$ Temp and Menthol conditions, respectively) was compared with that of $w^{1118}$ control flies bearing a single copy of the UASTRPM8 transgene, but no driver ( $n=30$ and 20 for the $\Delta$ Temp and Menthol conditions, respectively). CCAP-Gal4 $>1 \times$ UAS-TRPM 8 animals subjected neither to an $18^{\circ} \mathrm{C}$ temperature shift nor to menthol took $31 \pm 26$ min to expand their wings $(n=20)$. $B$, Bar graphs show the average duration of each behavioral phase for animals expressing $1 \times$ UAS-TRPM8 in N $\mathrm{N}_{\text {CAP }}$ ( $n=15$ ) compared with parallel control animals bearing the UAS-TRPM8 transgene, but no driver $(n=12)$. All animals were tested in the high-perturbation condition within 30 min of eclosion. Activation of $\mathrm{N}_{\text {CCAP }}$ using UAS-TRPM8 significantly shortened both phases I and II, but did not alter the duration of phase III. C, $N_{\text {CCAP }}$ activation causes rapid tanning, as indicated by these pictures of representative flies expressing $1 \times$ UAS-TRPM 8 in $\mathrm{N}_{\text {CCAP }}$, one of which received (right, $+\Delta$ Temp), and one of which did not receive (left, $-\Delta$ Temp), a 15 min temperature shift to $18^{\circ} \mathrm{C}$. Both flies were subjected in parallel to the high-perturbation condition and were photographed $3 \mathrm{~h}$ after the time of onset of the temperature pulse delivered to the experimental fly. All flies that received the temperature pulse $(n=20)$ expanded their wings and tanned, as indicated by the generally dark appearance of their cuticles, while control flies $(n=19)$ uniformly failed to expand their wings or tan and retained the whitish cuticle color seen at eclosion. $D, N_{\text {CCAP }}$ activation also causes rapid release of bursicon into the hemolymph. Hemolymph samples extracted from CCAP-Gal4 $>1 \times$ UAS-TRPM8 flies that either had (right, + ) or had not (left, - ) been subjected to a 15 min temperature shift to $18^{\circ} \mathrm{C}$ were analyzed by Western blot probed with an antibody to the bursicon $\alpha$-subunit. For each experimental condition, hemolymph was extracted from $>40$ flies that had been subjected to the highperturbation condition. Hemolymph was collected $1 \mathrm{~h}$ after the onset of the temperature shift or an equivalent time in unshifted controls. Molecular weight markers (in $\mathrm{kDa}$ ) are shown.

Elucidating which neurons mediate the decision to perch will also require further investigation. Although perching can be induced by the activation of $\mathrm{N}_{\mathrm{CCAP}}$ using UAS-TRPM8, $\mathrm{N}_{\mathrm{CCAP}}$ is clearly not normally required for terminating phase I, since its suppression does not affect perching. Also, the perching that does occur when $\mathrm{N}_{\text {CCAP }}$ is stimulated by UAS-TRPM8 is more tightly linked temporally with the initiation of expansion (i.e., phase III) than with the onset of $\mathrm{N}_{\mathrm{CCAP}}$ stimulation (i.e., the shift to $18^{\circ} \mathrm{C}$ ), suggesting that neurons downstream of $\mathrm{N}_{\mathrm{CCAP}}$ mediate phase I termination, most likely at the level of the motor networks underlying perch selection and expansion, as shown in Figure 6. Inhibition of one motor system by another has been demonstrated to explain such behavioral hierarchies in other cases, as in

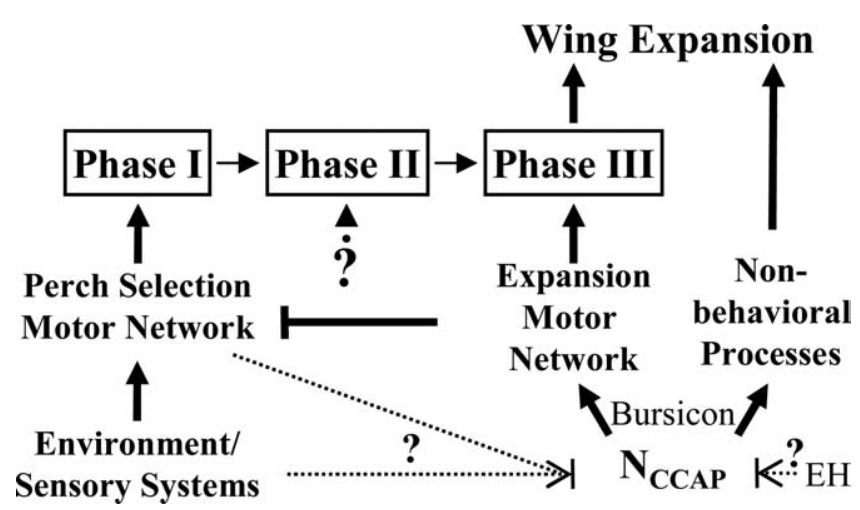

Figure 6. Proposed model for modulation of $\mathrm{N}_{\text {CCAP }}$ and posteclosion processes involved in wing expansion. Solid lines indicate interactions supported by the experimental evidence described here and elsewhere. Dotted lines indicate interactions that are either inferred or hypothesized. Our data demonstrate that phase I (i.e., perch selection) is regulated both by environmental factors and by the expansion motor network. Environmental inputs are depicted as positively driving perch selection, but it is equally possible that the perch selection program is endogenously active and inhibited by stimuli that signal a suitable environment for perching. $\mathrm{N}_{\text {CCAP }}$ activity is necessary and sufficient for activation of the expansion program, and, as indicated, also appears necessary for nonbehavioral processes (such as cuticle plasticization) that are required for wing expansion. Both effects are likely mediated by the release of the hormone bursicon, which is controlled by $\mathrm{N}_{\text {CCAP. }}$. The mechanisms of $\mathrm{N}_{\text {CCAP }}$ regulation may include either the perch selection motor network or environmental signals, although these are likely to act indirectly, as described more fully in the Discussion. Eclosion hormone (EH) is also likely to indirectly modulate $\mathrm{N}_{\text {CCAP }}$ activity, as described in the Discussion. The factors that regulate phase Il are unknown, but as mentioned in the Discussion, its duration may be governed in part by bursicon. Both the control and biological significance of this phase remain enigmatic and will have to be addressed by future experiments.

the dominance of feeding over withdrawal in mollusks (Davis, 1979).

\section{$\mathrm{N}_{\text {CCAP }}$ regulates the expansion program}

In contrast to perch selection, the expansion program shows no obvious environmental dependence, but it is strongly dependent on levels of $\mathrm{N}_{\mathrm{CCAP}}$ activity for its initiation. This is consistent with the known effects of bursicon, which is released by a subset of $\mathrm{N}_{\text {CCAP }}$ (Luan et al., 2006) and is required for both wing extensibility (Reynolds, 1976, 1977; Dai et al., 2008) and phase III behaviors (Baker and Truman, 2002). The probability of expansion occurring at all is likely to depend on whether the bursicon released into the CNS reaches a critical threshold, while the initiation of expansion most likely depends on the timing and/or rate of bursicon release. The rate of release should decline with $\mathrm{N}_{\mathrm{CCAP}}$ suppression, which will reduce the excitability of the bursiconexpressing neurons, and may account for both the prolongation of phase II under this condition and the appearance of graded wing expansion deficits. It is also possible that the timing of bursicon release was modulated by our manipulations of $\mathrm{N}_{\mathrm{CCAP}}$ if delay circuits intrinsic to $\mathrm{N}_{\mathrm{CCAP}}$ are responsible for initiating its secretion (see below).

Although it remains to be determined whether $\mathrm{N}_{\mathrm{CCAP}}$ participates in the decision to expand, it is clear that its activation drives all the behavioral and somatic processes necessary for wing expansion (see Fig. 6), placing $\mathrm{N}_{\mathrm{CCAP}}$ neurons high in the execution pathway of the decision and indicating that their function in wing expansion is command-like. Because this command-like function relies on bursicon and not direct synaptic activation, it differs from familiar command systems that mediate fast behavioral switches, such as those involved in defensive escape (Edwards et al., 1999), and more closely resembles systems in which hor- 
mones or neuromodulators elicit profound behavioral transitions such as those responsible for egg-laying in mollusks (Kupferman, 1967), stomatogastric ganglion modulation in crustacea (Hooper and Marder, 1984), and ecdysis in insects (Truman and Riddiford, 1970). Because of the technical difficulty involved, the ability of such systems to drive behavioral programs upon activation has rarely been demonstrated. However, as illustrated by the work presented here, the introduction of genetic techniques for in vivo neuronal activation should make such demonstrations increasingly possible.

\section{Coupling perch selection and expansion: insights from ecdysis}

The work presented here argues that the motor programs for perch selection and wing expansion have distinct regulatory mechanisms, but leaves unanswered the question of how the decisions to perch and expand are coupled: Specifically, the mechanism by which $\mathrm{N}_{\mathrm{CCAP}}$ is activated following perching remains to be clarified. As indicated in Figure 6, $\mathrm{N}_{\mathrm{CCAP}}$ could, in principle, receive input from either the perch selection motor network or from sensory processing pathways. Such input is likely to be indirect since perching and expansion are separated by a delay (i.e., phase II), which varies considerably in duration between individual animals and different conditions (see Figs. 2, 3). This delay period, during which bursicon secretion is initiated, may act, at least in part, to permit bursicon to plasticize the cuticle of the wing and body before expansion.

Behavioral transitions during ecdysis in both Drosophila and the hawkmoth Manduca sexta, are known to be regulated by inhibitory delay circuits (Zitnan and Adams, 2000; Fuse and Truman, 2002). In the case of eclosion hormone, the delay circuit temporally separates the somatic and behavioral aspects of the hormone's action and has been proposed to act as a control point for environmental modulation by light, which accelerates eclosion, perhaps by suppressing the inhibitory delay circuit (Baker et al., 1999; Truman, 2005). Interestingly, the modulation of eclosion by light is eliminated in animals lacking $\mathrm{N}_{\mathrm{CCAP}}$, supporting a role for these neurons in the inhibitory pathway (Park et al., 2003). Because ablation of the EH-expressing neurons causes wing expansion deficits, it is possible that in addition to modulating eclosion, EH may similarly modulate wing expansion by simultaneously upregulating excitability in both the bursiconexpressing neurons and in a delay circuit that inhibits them (McNabb et al., 1997; McNabb and Truman, 2008). By analogy to the action of $\mathrm{EH}$ on ecdysis, the latter circuit, which could include non-bursicon-expressing $\mathrm{N}_{\mathrm{CCAP}}$ neurons, would inhibit bursicon release until inhibition is alleviated by an environmentally mediated signal. Such a model might explain not only the wing expansion deficits observed in animals lacking EH-expressing neurons, but also the eventual wing expansion of animals kept in the high-perturbation condition. In these animals, run down in the delay circuit may trigger bursicon release before the decision to perch, as happens when $\mathrm{N}_{\text {CCAP }}$ is activated by UAS-TRPM8, and thus also account for their abbreviated phase II (see Fig. 2).

\section{$\mathrm{N}_{\text {CCAP }}$ function and behavioral adaptation}

The merits of this model, and others that invoke delay circuits, remain to be tested. It is interesting to note, however, that the mechanism(s) coupling environmental input to $\mathrm{N}_{\text {CCAP }}$ is likely to be added during metamorphosis, because the function of this network changes from an intrinsically regulated mediator of pupal ecdysis to an extrinsically modulated mediator of wing expansion (Park et al., 2003; Kim et al., 2006; Luan et al., 2006). Despite its broad phylogenetic conservation, this network also appears to be differentially regulated in different species, with CCAPexpressing neurons being direct targets of $\mathrm{EH}$ in many insects, but not Drosophila (Ewer and Truman, 1996). Such differential regulation may underlie, at least in part, species-specific differences in the coordination of wing expansion with adult emergence: In some insects, expansion is initiated at emergence without benefit of environmental input, and in others, like honeybees, it is initiated and completed before emergence. Investigation of the basis of these differences, and of the neural mechanisms that adapt $\mathrm{N}_{\mathrm{CCAP}}$ function to the individual developmental needs and life histories of different insects, should provide insight into general mechanisms of behavioral adaptation (Katz and HarrisWarrick, 1999).

In general, the work presented here demonstrates that the behavioral programs used by Drosophila to achieve wing expansion can serve as a simple and fruitful model for investigating networks underlying behavioral choice. Elucidation of the neuronal pathways that mediate the decisions to perch and expand the wings will shed light on the development and evolution of behavioral networks as well as their architecture and function. Indeed, the decision-making architecture outlined here for wing expansion may prove relevant to the type of instinctive behaviors, first described by Craig (1918), in which an environmentally sensitive "appetitive" phase is coupled to a "consummatory" phase consisting of a stereotyped, motor pattern. Finally, tools that permit one to manipulate decision-making in living, behaving animals, such as UAS-TRPM8, which we introduce here, clearly will play an essential role in mapping the neural networks underlying behavior in Drosophila and other animals.

\section{References}

Adamantidis AR, Zhang F, Aravanis AM, Deisseroth K, de Lecea L (2007) Neural substrates of awakening probed with optogenetic control of hypocretin neurons. Nature 450:420-U429.

Bainbridge SP, Bownes M (1981) Staging the metamorphosis of Drosophila melanogaster. J Embryol Exp Morphol 66:57-80.

Baker JD, Truman JW (2002) Mutations in the Drosophila glycoprotein hormone receptor, rickets, eliminate neuropeptide-induced tanning and selectively block a stereotyped behavioral program. J Exp Biol 205:2555-2565

Baker JD, McNabb SL, Truman JW (1999) The hormonal coordination of behavior and physiology at adult ecdysis in Drosophila melanogaster. J Exp Biol 202:3037-3048.

Cavener DR (1987) Comparison of the consensus sequence flanking translational start sites in Drosophila and vertebrates. Nucleic Acids Res 15:1353-1361.

Clark AC, del Campo ML, Ewer J (2004) Neuroendocrine control of larval ecdysis behavior in Drosophila: complex regulation by partially redundant neuropeptides. J Neurosci 24:4283-4292.

Colburn RW, Lubin ML, Stone DJ Jr, Wang Y, Lawrence D, D’Andrea MR, Brandt MR, Liu Y, Flores CM, Qin N (2007) Attenuated cold sensitivity in TRPM8 null mice. Neuron 54:379-386.

Cottrell CB (1962) General observations on the imaginal ecdysis of blowflies. Trans R Entomol Soc Lond 114:317-333.

Craig W (1918) Appetites and aversions as constituents of instincts. In: Foundations of animal behavior: classic papers with commentaries (Houck LD, Drickamer LC, eds), pp 276-292. Chicago: University of Chicago.

Dai L, Dewey EM, Zitnan D, Luo CW, Honegger HW, Adams ME (2008) Identification, developmental expression, and functions of bursicon in the tobacco hawkmoth, Manduca sexta. J Comp Neurol 506:759-774.

Davis WJ (1979) Behavioral hierarchies. Trends Neurosci 2:5-7.

Dewey EM, McNabb SL, Ewer J, Kuo GR, Takanishi CL, Truman JW, Honegger HW (2004) Identification of the gene encoding bursicon, an insect neuropeptide responsible for cuticle sclerotization and wing spreading. Curr Biol 14:1208-1213. 
Dhaka A, Murray AN, Mathur J, Earley TJ, Petrus MJ, Patapoutian A (2007) TRPM8 is required for cold sensation in mice. Neuron 54:371-378.

Dickson BJ (2008) Wired for sex: the neurobiology of Drosophila mating decisions. Science 322:904-909.

Edwards DH, Heitler WJ, Krasne FB (1999) Fifty years of a command neuron: the neurobiology of escape behavior in the crayfish. Trends Neurosci 22:153-161.

Ewer J (2005) Behavioral actions of neuropeptides in invertebrates: insights from Drosophila. Horm Behav 48:418-429.

Ewer J, Truman JW (1996) Increases in cyclic 3',5' -guanosine monophosphate (cGMP) occur at ecdysis in an evolutionarily conserved crustacean cardioactive peptide-immunoreactive insect neuronal network. J Comp Neurol 370:330-341.

Fraenkel G (1935) Observations and experiments on the blow-fly (Calliphora erythrocephala) during the first day after emergence. Proc Zool Soc Lond 87:893-904.

Fraenkel G, Hsiao C (1965) Bursicon, a hormone which mediates tanning of the cuticle in the adult fly and other insects. J Insect Physiol 11:513-556.

Fuse M, Truman JW (2002) Modulation of ecdysis in the moth Manduca sexta: the roles of the suboesophageal and thoracic ganglia. J Exp Biol 205:1047-1058.

Gammie SC, Truman JW (1997) Neuropeptide hierarchies and the activation of sequential motor behaviors in the hawkmoth, Manduca sexta. J Neurosci 17:4389-4397.

Holmes TC, Sheeba V, Mizrak D, Rubovszky B, Dahdal D (2007) Circuitbreaking and behavioral analysis by molecular genetic manipulation of neural activity in Drosophila. In: Invertebrate neurobiology (North G, Greenspan, RJ, eds), pp 19-52. Cold Spring Harbor, NY: Cold Spring Harbor Laboratory.

Hooper SL, Marder E (1984) Modulation of a central pattern generator by 2 neuropeptides, proctolin and FMRFamide. Brain Res 305:186-191.

Katz PS, Harris-Warrick RM (1999) The evolution of neuronal circuits underlying species-specific behavior. Curr Opin Neurobiol 9:628-633.

Kim YJ, Zitnan D, Galizia CG, Cho KH, Adams ME (2006) A command chemical triggers an innate behavior by sequential activation of multiple peptidergic ensembles. Curr Biol 16:1395-1407.

Kimura KI, Truman JW (1990) Postmetamorphic cell death in the nervous and muscular systems of Drosophila melanogaster. J Neurosci 10:403-411.

Kristan W, Gillette R (2007) Behavioral choice. In: Invertebrate neurobiology (North G, Greenspan, RJ, eds), pp 533-553. Cold Spring Harbor, NY: Cold Spring Harbor Laboratory.

Kupfermann I (1967) Stimulation of egg laying - possible neuroendocrine function of bag cells of abdominal ganglion of Aplysia californica. Nature 216:814-815

Luan H, Lemon WC, Peabody NC, Pohl JB, Zelensky PK, Wang D, Nitabach MN, Holmes TC, White BH (2006) Functional dissection of a neuronal network required for cuticle tanning and wing expansion in Drosophila. J Neurosci 26:573-584.

Luo CW, Dewey EM, Sudo S, Ewer J, Hsu SY, Honegger HW, Hsueh AJ (2005) Bursicon, the insect cuticle-hardening hormone, is a heterodimeric cystine knot protein that activates $G$ protein-coupled receptor LGR2. Proc Natl Acad Sci U S A 102:2820-2825.

Luo L, Callaway EM, Svoboda K (2008) Genetic dissection of neural circuits. Neuron 57:634-660.

McKemy DD, Neuhausser WM, Julius D (2002) Identification of a cold receptor reveals a general role for TRP channels in thermosensation. $\mathrm{Na}$ ture 416:52-58.

McNabb SL, Truman JW (2008) Light and peptidergic eclosion hormone neurons stimulate a rapid eclosion response that masks circadian emergence in Drosophila. J Exp Biol 211:2263-2274.

McNabb SL, Baker JD, Agapite J, Steller H, Riddiford LM, Truman JW
(1997) Disruption of a behavioral sequence by targeted death of peptidergic neurons in Drosophila. Neuron 19:813-823.

Miesenböck G, Kevrekidis IG (2005) Optical imaging and control of genetically designated neurons in functioning circuits. Ann Rev Neurosci 28:533-563.

Nagel G, Szellas T, Huhn W, Kateriya S, Adeishvili N, Berthold P, Ollig D, Hegemann P, Bamberg E (2003) Channelrhodopsin-2, a directly lightgated cation-selective membrane channel. Proc Natl Acad Sci U S A 100:13940-13945.

O'Brien MA, Taghert PH (1998) A peritracheal neuropeptide system in insects: release of myomodulin-like peptides at ecdysis. J Exp Biol 201:193-209.

Park JH, Schroeder AJ, Helfrich-Förster C, Jackson FR, Ewer J (2003) Targeted ablation of CCAP neuropeptide-containing neurons of Drosophila causes specific defects in execution and circadian timing of ecdysis behavior. Development 130:2645-2656.

Peier AM, Moqrich A, Hergarden AC, Reeve AJ, Andersson DA, Story GM, Earley TJ, Dragoni I, McIntyre P, Bevan S, Patapoutian A (2002) A TRP channel that senses cold stimuli and menthol. Cell 108:705-715.

Reid SNM, Fraenkel G, Friedman S (1987) Extrication, the primary event in eclosion, and its neural control in Sarcophaga-bullata. J Insect Physiol 33:481-486.

Reynolds SE (1976) Hormonal regulation of cuticle extensibility in newly emerged adult blowflies. J Insect Physiol 22:529-534.

Reynolds SE (1977) Control of cuticle extensibility in wings of adult manduca at time of eclosion - effects of eclosion hormone and bursicon. J Exp Biol 70:27-39.

Schroll C, Riemensperger T, Bucher D, Ehmer J, Völler T, Erbguth K, Gerber B, Hendel T, Nagel G, Buchner E, Fiala A (2006) Light-induced activation of distinct modulatory neurons triggers appetitive or aversive learning in Drosophila larvae. Curr Biol 16:1741-1747.

Suh GS, Ben-Tabou de Leon S, Tanimoto H, Fiala A, Benzer S, Anderson DJ (2007) Light activation of an innate olfactory avoidance response in Drosophila. Curr Biol 17:905-908.

Tinbergen N (2005) On aims and methods of ethology. (Reprinted from Zeitschrift für Tierpsychologie, Vol 20, p 410, 1963). Anim Biol 55:297-321.

Truman JW (2005) Hormonal control of insect ecdysis: endocrine cascades for coordinating behavior with physiology. In: Insect hormones, Vol 73 (Litwak G, ed), pp 1-30. Amsterdam: Academic.

Truman JW, Riddiford LM (1970) Neuroendocrine control of ecdysis in silkmoths. Science 167:1624-1626.

White BH, Osterwalder TP, Yoon KS, Joiner WJ, Whim MD, Kaczmarek LK, Keshishian H (2001) Targeted attenuation of electrical activity in Drosophila using a genetically modified $\mathrm{K}(+)$ channel. Neuron 31:699-711.

Wu Q, Wen T, Lee G, Park JH, Cai HN, Shen P (2003) Developmental control of foraging and social behavior by the Drosophila neuropeptide Y-like system. Neuron 39:147-161.

Yang CH, Belawat P, Hafen E, Jan LY, Jan YN (2008) Drosophila egg-laying site selection as a system to study simple decision-making processes. Science 319:1679-1683.

Zdarek J, Zavadilova J, Su J, Fraenkel G (1984) Post-eclosion behavior of flies after emergence from the puparium. Acta Entomol Bohem 81:161-170.

Zitnan D, Adams ME (2000) Excitatory and inhibitory roles of central ganglia in initiation of the insect ecdysis behavioural sequence. J Exp Biol 203:1329-1340.

Zitnan D, Kim YJ, Zitnanová I, Roller L, Adams ME (2007) Complex steroid-peptide-receptor cascade controls insect ecdysis. Gen Comp Endocrinol 153:88-96. 